\title{
A CHARACTERIZATION OF LOCALLY EUCLIDEAN SPACES
}

\author{
BY \\ O. G. HARROLD, JR.(1)
}

CHAPTER I

1. Introduction. This paper may be regarded as a continuation of Locally peripherally euclidean spaces are locally euclidean [6]. However, the exposition below is intended to be self-contained and complete. The chief accomplishment is a topological characterization of the $n$-dimensional sphere amongst compact metric spaces.

A set-theoretic problem that has been of interest for some time is that of characterizing the locally euclidean spaces among a more general class of topological spaces. In the one- and two-dimensional cases a possible candidate for the more general class of topological spaces has been the Peano spaces. An expository paper by Van Kampen in the first volume of the Duke Mathematical Journal summarizes some well-known results in this situation. In the higher-dimensional cases the results attained so far have had to avoid the difficulty that the Schoenflies theorem of plane topology does not extend in the most general form. One method in the case $n=3$ has been to assume the existence of a family of distinguished 2-spheres satisfying a system of five postulates and then prove that a sequence of partitionings exists that permits a homeomorphism between a 3-sphere and the topological space to be established [3], [6].

It turns out that the axiom system in [6] may be carried over more or less directly to the $n$-dimensional case. However, the proof that a compact metric continuum satisfying our axiom system is topologically the $n$-sphere rests on the observation that the combinatorial steps in [7] and [6] give one a scheme for approximating in a bi-uniform way certain subdivisions of the boundary of an $n+1$ simplex and the appropriately chosen subdivisions (or partitions) of the compact metric continuum $X$.

A brief outline of the proof that the axioms imply $X$ is $S^{n}$ may be helpful. A particular type of partitioning of an $(n-1)$-sphere is introduced in $\$ 2$ that can be extended to a partitioning of an open set of which the $(n-1)$-sphere is a boundary (§4). This determines a product structure in a "complex" that covers a neighborhood of the $(n-1)$-sphere. The word complex is put in quotes for the following

Received by the editors January 6, 1964.

(1) Research supported in part by the National Science Foundation Grant GP-211. 
reason. Each element of the complex in the domain space is a genuine euclidean cell and may be expressed as a topological product of cells of lower dimensions or as a "join" product. The corresponding element in the range space is not known to have a euclidean structure-that is just what is to be proved-but the intersection relations of the boundaries of the elements are indeed isomorphic to those of the corresponding elements of the domain.

Having given a homeomorphism ( $\S 8)$ from the boundary of an $n$-simplex to an element of our distinguished family of $(n-1)$-spheres $(\$ 7)$ and a columnar partition of a neighborhood of the boundary of the $n$-simplex, can this columnar partitioning be copied over in the range space? By the Properties 1,2,3, and 5 of the family $\mathscr{S}$ this turns out to be possible. In order to establish the desired homeomorphism of the $n$-simplex onto the closure of the open set in the range space of which the element of the family $\mathscr{S}$ is the boundary, iteration is called for. The linear structure of the $n$-simplex (\$6) provides us with a means of doing this in our domain space and, in fact, the iteration has a kind of uniformity. In the range space the Properties $1,2,3$, and 5 provide the possibility of iteration and Property 4 provides a needed means of securing uniformity $(\$ 10)$ since no linear structure is present to rely on.

It is a basic property of barycentric subdivisions of a euclidean $n$-simplex that if $d$ is the diameter of a simplex, each simplex of the $k$-barycentric subdivision has a diameter $\leqq(n /(n+1))^{k} d$. This implies that if (only) barycentric subdivisions are used in breaking up an $n$-dimensional cell into small pieces, then uniformity in size at successive stages is trivial. However, in our procedure below it seems necessary to shuttle back and forth between a barycentric subdivision and the dual subdivision. Hence the existence of a uniform system of subdivisions of a euclidean cell of the kind we need is not obvious.

The author wishes to thank the referee for helpful suggestions.

2. The systems $\mathscr{H}, \mathscr{A}$. In the following there will be occasion to use partitionings of the following variety of a topological space $W$.

Let $g_{1}, \cdots, g_{x}$ be disjoint open sets in $W$ such that $W$ is the closure of the union i.e., $W=\bigcup \bar{g}_{i}=\bigcup \bar{g}_{i}$.

In the cases of interest to us the set $g_{i}$ will be not only connected but also uniformly locally connected (0-ulc). Further, each $g_{i}=\operatorname{Int~} \mathrm{Cl} g_{i}$. Such decompositions have been called regular partitionings. A sequence $G_{1}, G_{2}, \cdots$ of regular partitionings is called a decreasing sequence provided each set in $G_{i+1}$ is a subset of some set in $G_{i}$ and the maximum diameter of the sets in $G_{i}$ approach zero with $1 / i$.

Let $U$ be an open set in a topological space so that $S=\bar{U} \backslash U$ is a topological $(n-1)$-sphere, the boundary of $U$. Let $\mathscr{K}_{S}$ be a simplicial subdivision of $S$ all of whose simplexes have a diameter $<\delta\left(\mathscr{K}_{S}\right)$, to be specified later. Let $K^{\prime}, K^{\prime \prime}, \cdots$ denote the first, second, $\cdots$ barycentric subdivisions of $\mathscr{K}$. Let $\tilde{K}$ be the dual of $K^{\prime}$. Let 


$$
\mathscr{H}=\left\{h_{1}, \cdots, h_{k_{n}}\right\}
$$

be the collection of open $(n-1)$-cells in $\tilde{K}$.

For any open subset $U$, denote the boundary by $F(U)=\bar{U} \backslash U$. If $\mathscr{K}_{s}$ is a subdivision of $S$ into a combinatorial $(n-1)$-sphere, then $F\left(h_{i}\right)=h_{i} \backslash h_{i}$ is a combinatorial $(n-2)$-sphere.

Suppose $\left(\gamma_{1}, \cdots, \gamma_{p}\right)$ is a subset of $\left(1,2, \cdots, k_{n}\right)$ such that ( $\square$ denoting the empty set) (a) $F\left(h_{\gamma_{1}}\right) \cap \cdots \cap F\left(h_{\gamma_{p}}\right) \neq \square, p>1$.

Then, by properties of the dual subdivision (see, for instance, Theorem 5.29 of [1]),

(b) $F\left(h_{\gamma_{1}}\right) \cap \cdots \cap F\left(h_{\gamma_{p}}\right)=d_{\gamma_{1} \ldots \gamma_{p}}(S)$

is a closed $(n-p)$-cell. If $x_{i}^{j}$ is the vertex inserted in the cell $\sigma_{i}^{j}$ of $\mathscr{K}$ in the formation of $K^{\prime}$, then

(c) $h_{i}=$ Int Cl St $x_{i}^{j}$, where the star is taken relative to $K^{\prime \prime}$. We call $h_{i}$ an element of 'Type $j+1$.

In order to avoid complications of notation the elements of Type $1, \cdots, n$ are ordered into a sequence so that if $i<j$ then $h_{i}$ has a Type $\leqq$ Type of $h_{j}$. It is supposed that only "straight" cells are employed relative to the given triangulation.

If a homeomorphism is given between $S$ and a simplex boundary $\dot{\sigma}^{n}$, a subdivision in either space determines one in the other. Suppose $a_{1}, \cdots, a_{k_{n}}$ form a partitioning of $\dot{\sigma}^{n}$ precisely analogous to the partitioning $h_{1}, \cdots, h_{k_{n}}$ of $S$. That is, the conditions (a), (b) and (c) above hold with $h_{i}$ replaced by $a_{i}$. Denote the collection $a_{1}, \cdots, a_{k}$ by $\mathscr{A}$.

The intersections of members of the family $\mathscr{A}$ form a cell complex $\mathscr{K}(\mathscr{A})$ where the incidence relations are determined by the partial order of set inclusion. A similar meaning is attached to $\mathscr{K}(\mathscr{B})$.

In Figure 1 a partitioning $\mathscr{A}=\left\{a_{1}, \cdots, a_{k_{n}}\right\}$ of the boundary $\dot{\sigma}^{n}$ of an $n$-simplex is shown. The partitioning $\mathscr{B}=\left\{b_{1}, \cdots, b_{k_{n}}\right\}$ of a neighborhood of $\dot{\sigma}^{n}$ in $\sigma^{n}$ is indicated (explained in $\S 6$ ).

Under a homeomorphism $f_{1}$ (§8) of $A=\dot{\sigma}^{n}$ onto $S=F(U)=\bar{U} \backslash U$, $\mathscr{H}=\left\{h_{1}, \cdots, h_{k_{n}}\right\}$ is a partitioning of $S$, where $h_{i}=f_{1}\left(a_{i}\right)$. We need a partitioning $\mathscr{G}=\left\{g_{1}, \cdots, g_{k_{n}}\right\}$ of a neighborhood of $S$ in $\bar{U}$ as described in the next paragraph.

The incidence relations between $\mathscr{A}$ and $\mathscr{B}$ stand in a certain simple relationship: If $\left(\gamma_{1}, \cdots, \gamma_{p}\right) \subset\left(1,2, \cdots, k_{n}\right)$ such that

then

$$
F\left(a_{\gamma_{1}}\right) \cap \cdots \cap F\left(a_{\gamma_{p}}\right) \neq \square,
$$

$$
F\left(a_{\gamma_{1}}\right) \cap \cdots \cap F\left(a_{\gamma_{p}}\right)=d_{\gamma_{1} \ldots \gamma_{p}}(A)
$$

is a closed $(n-p)$-cell and

$$
F\left(b_{\gamma_{1}}\right) \cap \cdots \cap F\left(b_{\gamma_{p}}\right)=d_{\gamma_{1} \ldots \gamma_{p}}(B)
$$

is a closed $(n-p+1)$-cell. We want to construct $\mathscr{G}$ so that if

$$
F\left(h_{\gamma_{1}}\right) \cap \cdots \cap F\left(h_{\gamma_{p}}\right)=d_{\gamma_{1} \ldots \gamma_{p}}(S)
$$

is an $(n-p)$-cell, then 


$$
F\left(g_{\gamma_{1}}\right) \cap \cdots \cap F\left(g_{\gamma_{p}}\right)=d_{\gamma_{1} \ldots \gamma_{p}}(U)
$$

is a closed $(n-p+1)$-cell.

By reference to Figure 1 we suspect that the linear structure of $\sigma^{n}$ may be used to define $\mathscr{B}$. In the case of $U$ no such linear structure is present and one must invent or discover how to define $\mathscr{G}$ properly. This may be accomplished as follows. In Figure 1 some sets are indicated that represent the element $a_{i}$ plus certain "flaps" or "wings," indicated by $a_{i}^{\prime}$. The system of sets $E(\mathscr{A})$ $=\left\{a_{1}^{\prime}, a_{2}^{\prime}, \cdots, a_{k_{n}}^{\prime}\right\}$ will be carried over to $S$ by $f_{1}$. By our axiom system (Chapter II) the images of these "flaps" may be bent or deformed into $U$. The intersection relations between the deformed flaps will provide the sets that will be the boundaries of the element of $\mathscr{G}$.

We now turn to the formal definition of $E(\mathscr{A})$.

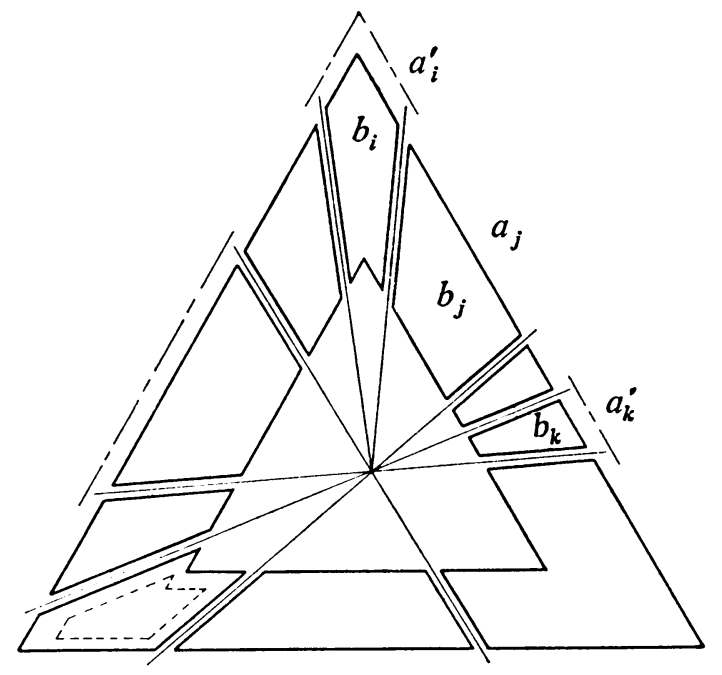

FIGURE 1

3. Definition of $E(\mathscr{A})$. Let there be $p_{1}, \cdots, p_{n}$ elements of Type $1, \cdots$, Type $n$, respectively, in the collection $\mathscr{A}=\left\{a_{1}, \cdots, a_{k_{n}}\right\}$. For $i=1,2, \cdots, k_{n}$ set $a_{i}=a_{i}(1)$. If $1 \leqq i \leqq p_{1}$, set $a_{i}^{\prime}=a_{i}(1)$. If $p_{1}+\cdots+p_{k-1}+1 \leqq i \leqq p_{1}+p_{2}+\cdots+p_{k}$, then, for each $2 \leqq \lambda \leqq k$, define

$$
a_{i}(\lambda)=\operatorname{Int} \mathrm{Cl}\left\{a_{i} \cup \underset{j \leqq p_{1}+\ldots+p_{k-1}}{\bigcup_{i}} \operatorname{St} \bar{a}_{i}(\lambda-1) \cap \bar{a}_{j}\right\},
$$

where St is taken relative to $\mathscr{K}^{(2 k)}$ and, finally,

$$
a_{i}^{\prime}=a_{i}(k) \text {. }
$$

By Corollary 14.3a of [2], it follows that $a_{i}^{\prime}$ may be regarded as an ( $\left.n-1\right)$-element, having been attained as the end result of a finite sequence of starring operations. 
That is, $a_{i}(2)$ is obtained from $a_{i}(1)$ by a finite number of applications of the fact that " $(n-1)$-element plus $(n-1)$-element is an $(n-1)$-element if the given pair meet precisely in an $(n-2)$-element common to their boundaries." Hence each of $a_{i}(1), a_{i}(2), \cdots, a_{i}(k)=a_{i}^{\prime}$ may be regarded as an element in the next combinatorial element of the sequence. If a combinatorial $(n-1)$-sphere contains $a_{i}^{\prime}$ as an element, the boundary of $a_{i}^{\prime}$ has the Schoenflies extension property (Theorem 14.2 of [2]).

Let $E(\mathscr{A})=\left\{a_{1}^{\prime}, \cdots, a_{k_{n}}^{\prime}\right\}$. Evidently $a_{i}^{\prime} \cap a_{j}^{\prime} \neq \square$ if and only if $\bar{a}_{i} \cap \bar{a}_{j} \neq \square$. Further, $a_{i}^{\prime} \cap a_{j}^{\prime}$ is then an $(n-1)$-element.

We call $E(\mathscr{A})$ the system of enlargements of $\mathscr{A}$.

4. The columnar partition $\mathscr{G}$. In this section we merely list the (desirable) properties of $\mathscr{G}$ without showing how to construct such sets. In general, $a, b, \cdots$ will denote rectilinear cells obtained by subdividing a simplex and $\mathscr{A}, \mathscr{B}, \cdots$ certain collections of such cells. The symbols $g, h, \cdots$ denote certain subsets of the range space and $\mathscr{G}, \mathscr{H}, \cdots$ will denote certain collections of such subsets. The sets $h$ will be defined in terms of a fixed homeomorphism of a simplex boundary $\dot{\sigma}$ to a set $S$. Thus there is an induced straightness for the sets in the collection $\mathscr{H}$. After the main result is proved it will be possible to speak of "straightness" of the sets in the collection $\mathscr{G}$.

Let $\mathscr{G}=\left\{g_{1}, \cdots, g_{k_{n}+1}\right\}$ be a collection of open sets that partition $U$ such that

(i) $F\left(g_{i}\right)$ is a topological $(n-1)$-sphere;

(ii) $S \cap F\left(g_{i}\right)=h_{i}, i=1, \cdots, k_{n}\left(h_{i}\right.$ as in $\left.\S 2\right), F\left(g_{k_{n}+1}\right) \cap F\left(g_{i}\right)$ is an $(n-1)$-disk;

(iii) $\bigcup_{1}^{k_{n}} \bar{g}_{i}$ is a neighborhood of $S$ in $\bar{U}$;

(iv) If $\left(\gamma_{1}, \cdots, \gamma_{n}\right)$ is a subset of $\left(1, \cdots, k_{n}\right)$ such that

then

$$
F\left(h_{\gamma_{1}}\right) \cap \cdots \cap F\left(h_{\gamma_{p}}\right)=d_{\gamma_{1} \ldots \gamma_{p}}(S) \text { (a closed }(n-p) \text {,-cell), }
$$

$$
F\left(g_{\gamma_{1}}\right) \cap \cdots \cap F\left(g_{\gamma_{p}}\right)=d_{\gamma_{1} \ldots \gamma_{p}}(U)
$$

is a closed $(n-p+1)$-cell.

The collection $\mathscr{G}=\left\{g_{1}, \cdots, g_{k_{n}+1}\right\}$ is called a columnar partitioning of $U$ generated by $\mathscr{K}_{\mathrm{s}}$.

Define $Z\left(h_{i}\right)$ to be the union of $h_{i}$ and all sets $d_{\gamma_{1} \ldots \gamma_{p}}(U)$ that meet $h_{i}$; then $Z\left(h_{i}\right)$ is the star of a cell in $F\left(g_{i}\right)$ under appropriate subdivision. Hence, defining

(v) $\mathcal{O}_{i}(U)=$ Closure of $F\left(g_{i}\right) \backslash Z\left(h_{i}\right)$,

then $\mathcal{O}_{i}(U)$ is an $(n-1)$-cell by Theorem 14.2 of [2]. Also,

(vi) $\mathcal{O}_{\gamma_{1} \ldots \gamma_{p}}(U)=\mathcal{O}_{\gamma_{1}} \cap \mathcal{O}_{\gamma_{2}} \cap \cdots \cap \mathcal{O}_{\gamma_{p}}$

is an $(n-p)$-cell.

5. Layers of columnar partitions. Suppose $\mathscr{G}=\left\{g_{1}, \cdots, g_{k_{n}+1}\right\}$ is a columnar partition of $U$ generated by $\mathscr{K}_{S}$. Now $F\left(g_{k_{n}+1}\right)=S_{1}$ is a topological $(n-1)$ sphere and any simplicial subdivision of $\mathscr{K}_{S_{1}}$ of $S_{1}$ induces a columnar partition 
of $U_{1}=g_{k_{n}+1}$. Let $\mathscr{G}_{1}=\left\{g_{1}^{\prime}, \cdots, g_{k_{u}^{\prime}+1}^{\prime}\right\}$ be such a partition of $U_{1}$. Then $\left\{g_{1}, \cdots, g_{k_{n}}, g_{1}^{\prime}, \cdots, g_{k_{n}+1}^{\prime \prime}\right\}$ is called a 2 -fold layer of columnar partitions of $U$. The extension to an $m$-fold layer of columnar partitions of $U$ is immediate

$$
\mathscr{G}, \mathscr{G}_{1}, \cdots, \mathscr{G}_{m-1},
$$

where the element constituting the "interior" of $U_{k}$ is decomposed into the elements making up $\mathscr{G}_{k+1}$ (putting $\mathscr{G}_{0}=\mathscr{G}$ and $U_{0}=U$ ).

Comment. In the proof below that a compactum $X$ that admits a family $\mathscr{S}$ is the $n$-sphere, it is sufficient to follow the details of the construction in the special case that the layer of columnar partitions of $U$ be of single thickness rather than an $m$-fold layer, $m>1$. In the proof that such a family $\mathscr{S}$ exists for $S^{n}$ it seems essential that $m$-fold layers be considered, $m \geqq 1$.

It will be shown in Chapter II how to construct layers of columnar partitions and in $\$ 9$ we prove properties (i)-(vii) may be attained.

(vii) Let $B$ be the unit $n$-ball and $A=\dot{B}$. Let $U$ be an open subset of a space $X$ such that $S=\bar{U} \backslash U$ is an $(n-1)$-sphere. Let $f$ be a homeomorphism of $A$ onto $S$ and $\mathscr{A}=\left\{a_{1}, \cdots, a_{p}\right\}$ a columnar partitioning of $\mathscr{A}$. Then $f$ induces a columnar partition $\mathscr{H}$ of $S, \mathscr{H}=\left\{h_{1}, \cdots, h_{p}\right\}$, by $h_{i}=f\left(a_{i}\right)$. Let $\mathscr{G}=\left\{g_{1}, \cdots, g_{P+1}\right\}$ be a 1 -fold layer of columnar partitions of $U$ generated by $\mathscr{H}$. Then there exists an extension $\bar{f}$ of $f$ with the following properties,

such that

$$
\bar{f}: A \times[0,1] \backslash \bigcup_{i=1}^{p} a_{i} \times(0,1) \rightarrow \bigcup_{i=1}^{p+1} F\left(g_{i}\right),
$$

for each $a \in \mathscr{A}$;

$$
\bar{f}(a, 0)=f(a)
$$

and

$$
\bar{f}\left[\operatorname{Bd}\left(\bar{a}_{i} \times[0,1]\right)\right]=F\left(g_{i}\right), \quad i \leqq p,
$$

$$
\bar{f}(A \times 1)=F\left(g_{p+1}\right) .
$$

Actually, one can show that property (vii) is a consequence of the definition of columnar partitions. Therefore, the reader may take this as a part of the definition.

6. Uniform partitions, the system $\mathscr{B}$. We call any partition of $U \eta$-uniform if and only if for each $g_{i}$ of the partition, diameter $\left(g_{i}\right) /$ diameter $U<\eta$.

Let $R$ be an $n$-dimensional, closed, bounded polyhedral region in $E^{n}$ that is star-like with respect to $O$. That is, any ray from $O$ meets the boundary $\dot{R}$ of $R$ in a single point. Let $\mathscr{K}$ be a simplicial subdivision of $\dot{R}$ into convex cells. On each ray $O P, P \in \dot{R}$, let $Q$ divide the segment $O P$ in the ratio 3: 8. Let $R_{1}$ denote the set of all points for which the ratio is less than $3: 8$ and let $\dot{R}_{1}$ denote the boundary of this region. The set $\dot{R}_{1}$ is clearly homeomorphic to $\dot{R}$ and the region bounded by $\dot{R} \cup \dot{R}_{1}$ is decomposed in an obvious way into cells $\zeta=\sigma \times[3 / 11,1]$, where $\sigma \in \mathscr{K}$. Each $\zeta$ is convex. Since $Q P / O P=8 / 11=.7272$, certainly daimeter $\zeta$ 
$<(3 / 4)$ (diameter $R)$ if the mesh of $\mathscr{K}$ is small enough. Note that diameter $R_{1}$ $<(3 / 4)$ (diameter $R)$.

There is thus a uniform partition of $R$ for $\eta=3 / 4$ into the sets whose elements are $\left\{R_{1}\right\} \cup\{\zeta\}$. If each of these elements is a star-like region, this process may be iterated to find a sequence of partitionings of $R$

$$
R, R_{\alpha_{1}}, R_{\alpha_{1}, \alpha_{2}}, R_{\alpha_{1}, \alpha_{2}, \alpha_{3}}, \cdots
$$

so that $R_{\alpha_{1}, \alpha_{2}} \subset R_{\alpha_{1}}$ and, for $\alpha_{1}, \cdots, \alpha_{n-1}$ fixed,

$$
\bigcup \bar{R}_{\alpha_{1} \ldots \alpha_{n}}=\bar{R}_{\alpha_{1} \ldots \alpha_{n-1}}
$$

and diameter $R_{\alpha_{1} \ldots \alpha_{n}}<(3 / 4)\left(\right.$ diameter $\left.R_{\alpha_{1} \ldots \alpha_{n-1}}\right)$.

Hence

$$
\bigcap_{n=1}^{\infty} \bar{R}_{\alpha_{1} \ldots \alpha_{n}}=p\left(\alpha_{1}, a_{2}, \cdots\right),
$$

for a fixed sequence $\alpha_{1}, \alpha_{2}, \cdots$, is a point.

Now if $S_{\alpha_{1} \ldots \alpha_{n}}$ is any region that is very near the region $R_{\alpha_{1} \ldots \alpha_{n}}$ in the sense of Hausdorff distance, then the diameter of $S_{\alpha_{1} \ldots \alpha_{n}}$ will be very near that of $R_{\alpha_{1} \ldots \alpha_{n}}$. Hence for any fixed $n$ and $\alpha_{1}, \cdots, \alpha_{n}$ given, we may subdivide $\dot{R}_{\alpha_{1} \ldots \alpha_{n}}$ sufficiently finely that, when we form the associated dual complex and the corresponding columnar partitionıng, diameter $S_{\alpha_{1} \ldots a_{n}}<(3 / 4)$ (diameter $R_{\alpha_{1} \ldots z_{n-1}}$ ).

We noted that $\zeta=\sigma \times[3 / 11,1]$ is convex for $\sigma \in K$. What is more important for the construction below is that if $\sigma_{i}$ is an $(n-1)$-element in $\widetilde{K}(\mathscr{A})$, then $\sigma_{i} \times[3 / 11,1]$ need not be convex, but it is star-like with respect to any point on $x_{i}^{0} \times[3 / 11,1]$, where $x_{i}^{0}$ is the vertex inserted in a vertex of $K$ in the formation of $\sigma_{i}$.

Let $P$ belong to the boundary of the cell $\sigma_{i} \times[3 / 11,1]$. Now $\sigma_{i}$ is a union of $(n-1)$-cells of $K^{\prime \prime}$ having $x_{i^{\prime}}^{0}$ as a vertex. Suppose $P$ lies in the simplex $\sigma_{i}^{n-1}$ of $K$ and that $q_{0}, \cdots, q_{n-1}$ are barycentric coordinates for $\sigma_{i}^{n-1}$. The simplexes of $\tilde{K}$ of dimension $n-1$ that lie in $\sigma_{i}^{n-1}$ and have $x_{i^{\prime}}^{0}$ as a vertex fall into $\sigma_{i}$. The points of these simplexes inherit barycentric coordinates from $\sigma_{i}^{n-1}$. Hence each point of $\sigma_{i} \times[3 / 11,1]$ may be given coordinates $\left(q_{0}, \cdots, q_{n-1}, t\right)$. If $Q$ is any point of $x_{i}^{0} \times[3 / 11,1]$, all points of the segment $P Q$ lie in $\sigma_{i} \times[3 / 11,1]$.

\section{ChaPTER II}

7. The space $X$. Let $X$ be a compact metric space such that $X$ contains a least two points and each point $p \in X$ has a local basis of open sets $\{U\}$ whose boundaries are members of a family $\mathscr{S}$ of subsets of $X$ satisfying the following requirements.

1. Separation Axiom. Each $S \in \mathscr{S}$ is a topological $(n-1)$-sphere that separates $X$ irreducibly into exactly two components. 
2. Deformation Axiom. If $D$ is a topological ( $n-1)$-cell, $D \subset S, S \in \mathscr{S}, U$ a component of $X \backslash S, V$ an open set containing Int $D$, then there is an isotopy of the identity map, the identity on $X \backslash V$, such that $F_{t}($ Int $D) \subset U$ for $0<t$, where $F_{t}(x), 0 \leqq t \leqq 1$, is a function realizing the isotopy.

3. Union Axiom. If $D_{1}$ and $D_{2}$ are topological $(n-1)$-cells, each a subset of some element of $\mathscr{S}, D_{1} \cap D_{2}=\partial D_{1} \cap \partial D_{2}, D_{i} \subset S_{i} \in \mathscr{S}$ and $S_{1} \cap S_{2}$ contains an annulus $A$ such that one component of $\partial A=S=\partial D_{1} \cap \partial D_{2}$, then $D_{1} \cup D_{2}=S^{\prime} \in \mathscr{S}$.

4. Contraction Axiom. There is a number $\eta, 0<\eta<1$, such that if $S \in \mathscr{S}, U$ is a component of $X \backslash S$, and $\mathscr{K}$ is a simplicial subdivision of $S$ of sufficiently small mesh, then to each layer of columnar partitionings $g_{1}, \cdots, g_{p}$ of $U$ of sufficiently many layers generated by $\mathscr{K}$ with $F\left(g_{i}\right) \in \mathscr{S}$ there is a homeomorphism $t$ of $\bar{U}$ on $\bar{U}$, the identity on $S$ such that if $g_{i}^{\prime}=t\left(g_{i}\right)$, then

$$
\operatorname{diam} g_{i}^{\prime}<\eta \operatorname{diam} U \text {. }
$$

5. Maximality Axiom. If $S \in \mathscr{S}$ and $t$ is a homeomorphism of $X$ on itself, then $t(S) \in \mathscr{S}$.

The following are easily proved consequences of the existence of a local basis at each point with the boundaries of the open sets being members of $\mathscr{S}$ (see Appendix):

(1) $X$ is connected;

(2) $X$ is locally connected.

The principal result to be proved below is

THEOREM. Let $X$ be a compact metric space containing at least two points such that each point has a local basis consisting of open sets whose boundaries are members of the family $\mathscr{S}$ satisfying the Separation, Deformation, Union, Contraction and Maximality Axioms. Then $X$ is homeomorphic to the $n$-sphere. Conversely, the $n$-sphere $S^{n}$ admits such a family $\mathscr{S}$ (Chapter III).

8. The homeomorphism $f_{1}$ and its deformations used to produce $\mathscr{G}$. Let $B$ be an $n$-dimensional cell in $E^{n}$ with a polyhedral boundary $\dot{B}=A$. We suppose $B$ is star-like with respect to the origin. One could require convexity of $B$ but since this property will not be preserved under the columnar partitions of $\S 4$, there is little point in insisting on it at this stage.

The topological space $X$ is supposed to have a local basis at each point consisting of open sets whose boundaries are elements of the family $\mathscr{S}$ described in $\S 7$.

In order to show that $X$ is an $n$-dimensional topological sphere let $p$ be a point of $X$, and $U$ an open set containing $p$ whose boundary $S \in \mathscr{S}$. It is proposed to show that $\bar{U}$ and $B$ are homeomorphic.

According to the Axiom of Contraction, if there exists a layer of columnar partitions of $U$, then a homeomorphism $t$ of $\bar{U}$ on $\bar{U}$ exists so that the points 
of $S=\bar{U} \backslash U$ are fixed and the image of any element of the layer of partitionings has a diameter less than $\eta$ times the diameter of $U$. In the following we describe in detail how the first layer in the layer of columnar partitions is produced. By iteration a sufficient number of layers is produced so that the Axiom of Contraction may be applied.

Any homeomorphism of $A$ on $S$ induces an isomorphism between a given partitioning $\mathscr{A}$ of $A$ and $\mathscr{H}$ of $S$ according to the rule: If $\mathscr{A}=\left\{a_{1}, \cdots, a_{k_{n}}\right\}$, then $\mathscr{H}=\left\{h_{1}, \cdots, h_{k_{n}}\right\}$, where $h_{i}$ and $a_{i}$ correspond under the homeomorphism.

Let $\mathscr{B}=\left\{b_{1}, \cdots, b_{k_{n}+1}\right\}$ be a $3 / 4$-uniform columnar partition of $B$ induced by $\mathscr{A}$ (see $\S 4$ ). Then we must show how to construct a partition $\mathscr{G}$ of $U$ induced by $\mathscr{H}$ so that $\mathscr{A}$ and $\mathscr{H}$ are isomorphic and $\mathscr{B}$ and $\mathscr{G}$ are isomorphic.

The columnar partitioning $\mathscr{B}$ of $B$ could be produced directly via the linear structure of $B$ without any need or reference to a system of enlargements $E(\mathscr{A})$. However, the system of enlargements $E(\mathscr{H})$ is necessary in order to define the columnar partition $\mathscr{G}$ of $\bar{U}$. Further, the desired bi-uniform correspondence can only be established it we anticipate the extension problem in the iteration step and in order to accomplish this extension we must obtain $E(\mathscr{H})$ via $E(\mathscr{A})$, wherein the desired extensions are possible.

Suppose $f_{1}$ is any homeomorphism of $\dot{B}$ on $S$. Then $h_{i}=f_{1}\left(a_{i}\right)$ determines a system $\mathscr{H}$ whose elements are subsets of $S$ and whose boundaries have isomorphic intersection properties to those of the boundaries of the elements in $\mathscr{A}$.

To prove that a $\mathscr{G}$ exists that is properly related to $\mathscr{H}$, that is, that satisfies conditions (i)-(vi) of $\S 4$, it will be convenient to start with a system $E(\mathscr{H})$ of enlargements of the elements of the system $\mathscr{H}$.

By means of the system $E(\mathscr{H})$ of enlargements of the system $\mathscr{H}$ of subsets of $S$, the homeomorphism $f_{1}$, and repeated use of the axioms for $\mathscr{S}$, the system $\mathscr{G}$ that constitutes a columnar partitioning of $U$ will be defined.

Step 1. By the Deformation Axiom and the fact that $h_{1}^{\prime}$ is an $(n-1)$-cell that is a subset of $S$ (an element of $\mathscr{S}$ ), there is a homeomorphism $l_{i}$ of $X$ on $X$ that pushes $h_{i}^{\prime}$ into $U$ and moves points an arbitrarily small amount. Since $h_{i}^{\prime} \cap h_{j}^{\prime}=\square$ if $h_{i}$ and $h_{j}$ are of the same Type, it is an elementary matter to define in terms of $l_{1}, l_{2}, \cdots, l_{p_{1}}$ a homeomorphism $G_{0}$ of $X$ on $X$ that pushes each $h_{i}^{\prime}$ into $U$ simultaneously, where $h_{i}$ is an element of Type 1. If $\delta_{1}=\min \rho\left[h_{1}^{\prime}, h_{j}^{\prime}\right], h_{i}, h_{j}$ of Type 1 and $\eta_{1}=\min \left(\varepsilon / 3 n, \delta_{1} / 3\right)$, we may suppose $G_{0}$ moves no point of $X$ as much as $\eta_{1}$.

The components of $U \backslash G_{0}(S)$ whose boundaries meet $\bigcup h_{i}^{\prime}, h_{i}$ of Type 1, are denoted $g_{1}, \cdots, g_{p_{1}}$. This family is the first subcollection of sets needed in the definition of $\mathscr{G}$ (see $\$ 4$ ).

Property (i) holds since $F\left(g_{i}\right)=h_{i}^{\prime} \cup G_{0}\left(h_{i}^{\prime}\right)$.

Property (ii) holds, namely $\bar{g}_{i} \cap S=h_{i}^{\prime}=h_{i}$, since $h_{i}$ is of Type 1 .

Property (iii) is inoperative, and

Property (iv) holds by definition of $\eta_{1}$. 
In order that the procedures may be iterated we summarize:

The homeomorphism $G_{0}$ exists by the Axiom of Deformation. By the Axiom of Maximality, $G_{0}(S) \in \mathscr{S}$. To see that $F\left(g_{i}\right) \in \mathscr{S}$ note that $h_{i}^{\prime} \subset S$ and $G_{0}(S) \in \mathscr{S}$. Hence $h_{i}^{\prime}$ and $G_{0}\left(h_{i}^{\prime}\right)$ correspond to $D_{1}$ and $D_{2}$ in the Union Axiom. Taking $S$ and $G_{0}(S)$ as $S_{1}$ and $S_{2}, S_{1} \cap S_{2}$ contains an annulus $A$ as required and hence $D_{1} \cup D_{2}=F\left(g_{i}\right) \in \mathscr{S}$.

Step 2. Consider the sets $G_{0}\left(h_{i}^{\prime}\right)$, where $h_{i}^{\prime}$ is of Type 2. Let $\delta_{2}$ $=\min \left(\rho\left[G_{0}\left(h_{i}^{\prime}\right), G_{0}\left(h_{j}^{\prime}\right)\right], h_{i}, h_{j}\right.$ of Type $2, \rho\left[G_{0}\left(h_{i}\right), G_{0}\left(h_{j}\right)\right], h_{i}$ of Type 1, $h_{j}$ of Type 2 and $\left.h_{i} \cap h_{j}=\square\right)$. Define $\eta_{2}=\min \left(\varepsilon / 3 n, \delta_{2} / 3\right)$. An open subset $V_{1}$ of $G_{0}(S)$ is defined by

$$
V_{1}=\bigcup G_{0}\left(h_{i}^{\prime}\right), \quad h_{i} \text { of Type } 2 .
$$

As before there is a space homeomorphism $G_{1}$ with $G_{1}$ pushing $V_{1}$ into $G_{0}(U)$ (the interior of $G_{0}(S)$ ) in such a way that the points of $G_{0}(S) \backslash V_{1}$ remain fixed and each point of $X$ is moved no more than $\eta_{2}$. The components of $G_{0}(U) \backslash G_{1} G_{0}(S)$ whose boundaries meet $V_{1}$ define the second set of $g$ 's needed in the construction of $\mathscr{G}$.

If $g_{i}$ is an element of Type 2 (i.e., the corresponding $h_{i}$ of Type 2), then $F\left(g_{i}\right)$ is the union of $G_{0}\left(h_{i}^{\prime}\right)$ and $G_{1} G_{0}\left(\bar{h}_{i}^{\prime}\right)$, hence (i) holds. Condition (ii) has two parts. The second part is inoperative until $g_{k_{n}+1}$ has been defined. To test the first part, note that $S \cap F\left(g_{i}\right)=S \cap G_{0}\left(\bar{h}_{i}\right)=S \cap h_{i}=h_{i}$. Condition (iii) does not apply. Condition (iv) is considered in two cases according as $h_{\gamma_{1}}$ and $h_{\gamma_{2}}$ are both of Type 2 or not. In the former case, the definition of $\delta_{2}$ shows $\bar{g}_{\gamma_{1}} \cap \bar{g}_{\gamma_{2}}=\square$. If $h_{\gamma_{1}}$ is of Type $1, h_{\gamma_{2}}$ is of Type 2 and $h_{\gamma_{1}} \cap h_{\gamma_{2}}=\square$, again the definition of $\eta_{2}$ shows that $\bar{g}_{\gamma_{1}} \cap \bar{g}_{\gamma_{2}}=\square$. If $h_{\gamma_{1}} \cap h_{\gamma_{2}} \neq \square$, then $h_{\gamma_{1}}^{\prime} \cap h_{\gamma_{2}}^{\prime}$ is an $(n-1)$-disk, namely, the star of $h_{\gamma_{1}} \cap h_{\gamma_{2}}$ in $h_{\gamma_{1}}$ relative to $(\widetilde{K})^{\prime \prime}$. Then $\bar{g}_{\gamma_{1}} \cap \bar{g}_{\gamma_{2}}$ is precisely the image of this disk under $G_{0}$, which is labelled $d_{\gamma_{1} \gamma_{2}}(U)$. The collection of $g$ 's of Types 1 and 2 satisfy all the conditions (i)-(iv) that apply. As before, each $F\left(g_{i}\right) \in \mathscr{S}$ and $G_{1} G_{0}(S) \in \mathscr{S}$.

Step $k$. Consider the sets $G_{k-2} \cdots G_{0}\left(h_{i}^{\prime}\right)$, where $h_{i}$ is of Type $k$. Let $\delta_{k}=\min \left(\rho\left[G_{k-2} \cdots G_{0}\left(h_{i}^{\prime}\right), G_{k-2} \cdots G_{0}\left(h_{j}^{\prime}\right)\right], h_{i}, h_{j}\right.$ of Type $k$,

$$
\rho\left[G_{k-2} \cdots G_{0}\left(h_{i}\right), G_{k-2} \cdots G_{0}\left(h_{j}\right)\right],
$$

$h_{j}$ of Type $<k$ and $\left.h_{i} \cap \bar{h}_{i}=\square\right)$. Define $\eta_{k}=\min \left(\varepsilon / 3 n, \delta_{k} / 3\right)$. An open subset of $G_{k-2} \cdots G_{0}(S)$ is defined by

$h_{i}$ of Type $k$.

$$
V_{k-1}=\bigcup G_{k-2} \cdots G_{0}\left(h_{i}^{\prime}\right)
$$

Again by the Axiom of Deformation, a space homeomorphism $G_{k-1}$ of $X$ on $X$ exists that pushes $V_{k-1}$ into $G_{k-2} \cdots G_{0}(U)$, is the identity on $G_{k-2} \cdots G_{0}(S) \backslash V_{k-1}$ and moves no point more than $\eta_{k}$. The components of $G_{k-2} \cdots G_{0}(U) \backslash G_{k-1} \cdots G_{0}(S)$ 
whose boundaries meet $V_{k-1}$ define the $k$ th set of $g$ 's needed in the construction of $\mathscr{G}$. The collection $g_{1}, \cdots, g_{p_{k}}$ satisfies condition (i).

It has already been observed that $g_{1}, \cdots, g_{p_{k-1}}$ satisfy (i) and the boundaries of the elements introduced at the $k$ th stage are each of the form $G_{k-2} \cdots G_{0}\left(h_{i}^{\prime}\right) \cup G_{k-1} G_{k-2} \cdots G_{0}\left(h_{i}^{\prime}\right)$. Hence (i) holds. Also, (ii) holds since for any $p_{1}+\cdots+p_{k-1}<i \leqq p_{1}+p_{2}+\cdots+p_{k}, G_{k-2} G_{k-3} \cdots G_{0}\left(h_{i}\right)=h_{i}, \bar{g}_{i} \cap S=h_{i}$. To see that (iv) holds, suppose that $h_{\gamma_{1}} \cap \cdots \cap \bar{h}_{\gamma_{j}}=[]$. The definition of $\delta_{k}$ implies that $\bar{g}_{\gamma_{1}} \cap \cdots \cap \bar{g}_{\gamma_{j}}=\square$. If on the other hand, $h_{\gamma_{1}} \cap \bar{h}_{\gamma_{2}} \cap \cdots \cap \bar{h}_{\gamma_{j}}=d_{\gamma_{1} \ldots \gamma_{j}}(S)$, a topological $(n-j)$-cell, we may suppose only one of $h_{\gamma_{1}} \cdots h_{\gamma_{j}}$ is of Type $k$, say $h_{\gamma_{j}}$. Then $h_{\gamma_{1}} \cap \cdots \cap h_{\gamma_{j-1}}=d_{\gamma_{1} \ldots \gamma_{j-1}}(S)$ is an $(n-j+1)$-cell and, by induction, $\bar{g}_{\gamma_{1}} \cap \cdots \cap \bar{g}_{\gamma_{j-1}}=d_{\gamma_{1} \ldots \gamma_{j-1}}(U)$ is a closed $(n-j+2)$-cell. The boundary of $d_{\gamma_{1} \ldots \gamma_{j-1}}(U)$ is an $(n-j+1)$-sphere that contains the $(n-j+1)$-cell $h_{\gamma_{1}} \cap \cdots \cap h_{\gamma_{j-1}}$. The map $G_{k-1}$ pushes $G_{k-2} \cdots G_{0}(U)$ away from $V_{k-1}$ and the components of $G_{k-2} \cdots G_{0}(U) \backslash G_{k-1} \cdots G_{0}(S)$ that have boundaries meeting the $h$ 's of Type $k$ are the set of $g$ 's of Type $k$. Each component of the star of $d_{\gamma_{1} \ldots \gamma_{j-1}}(S)$ in the boundary of $d_{\gamma_{1} \ldots \gamma_{j-1}}(U)$ not already in $d_{\gamma_{1} \ldots \gamma_{j-1}}(S)$ belongs to some $F\left(g_{i}\right)$, i.e., to some $\bar{g}_{i}$ of Type $k$. But such components are $(n-j+1)$-elements in the boundary of $d_{\gamma_{1} \ldots y_{j-1}}(U)$, i.e., an $(n-j+1)$-disk, and common to the boundaries of a $j$-tuple of $g$ 's.

Finally, at the completion of step $k=n$, (i) and (iv) hold as above. By construction, the first part of (ii) holds. Noting that $G_{i-1} \cdots G_{0}(S)$ is an element of $\mathscr{S}$, $g_{k_{n}+1}$ is defined as the component of $X \backslash G_{n-1} \cdots G_{0}(S)$ not meeting any of $g_{1}, \cdots, g_{k_{n}}$. From the definition of $\mathcal{O}_{i}(\$ 4)$ we see that the second part of (ii) holds. Condition (iii) holds because the system of enlargements $E(\mathscr{H})$ forms an open cover of $S$ and each point of $S$ has a neighborhood that is pushed into $U$ by one of $G_{0}, G_{1} G_{0}, \cdots G_{n-1} \cdots G_{0}$.

9. Extension of $f_{1}$ to the $(n-1)$-skeleton of $\mathscr{B}$. The construction of $\mathscr{G}=\left\{g_{1}, \cdots, g_{k_{n}+1}\right\}$ has been made $(\S 7$ and $\S 8)$ so that $\mathscr{B}=\left\{b_{1}, \cdots, b_{k_{n}+1}\right\}$ and $\mathscr{G}$ are isomorphic under the relation of preservation of non-null intersection of closures of corresponding collections. This implies that the $k$-skeleton of $\mathscr{B}$ and the $k$-skeleton of $\mathscr{G}$ are isomorphic. The question now arises as to the possibility of finding a homeomorphic extension of $f_{1}$ between the corresponding elements of the $k$-skeleton of $\mathscr{B}$ and that of $\mathscr{G}$.

To see that this may be accomplished let us recall that the homeomorphism $f_{1}$ was used in defining $\mathscr{H}=\left\{h_{1}, \cdots, h_{k_{n}}\right\}$ by the rule $h_{i}=f_{1}\left(a_{i}\right)$. Suppose we construct a system of enlargements of $\mathscr{A}=\left\{a_{1}, \cdots, a_{k_{n}}\right\}$ and then take the corresponding images of the elements of $E(\mathscr{A})=\left\{a_{1}^{\prime}, \cdots, a_{k_{n}}^{\prime}\right\}$ as the elements of $E(\mathscr{H})$ $=\left\{h_{1}^{\prime}, \cdots, h_{k_{n}}^{\prime}\right\}$. Since the cells of $E(\mathscr{A})$ may be regarded as "straight" in the sense of a proper triangulation of $\dot{B}$, the cells making up the faces of $g_{i}$ may be regarded as "straight" relative to the induced nerve of $\mathscr{G}$. 
The function $f_{1}$ furnishes the definition of a map $T_{k}$ of the $k$-skeleton of $\mathscr{K}(B)$ to $\mathscr{K}(U)$ when restricted to $\mathscr{K}(A)$ for each $k=0, \cdots, n-1$. The symbols $\mathscr{K}(\mathscr{A})$, etc., are defined in $\S 2$.

To extend $T_{0}$ from $\mathscr{K}(A)$ to $\mathscr{K}(B)$, suppose $\left(\gamma_{1}, \cdots, \gamma_{n}\right)$ is a subcollection of $\left(1, \cdots, k_{n}\right)$ such that $d_{\gamma_{1} \ldots \gamma_{n}}(A)$ exists (and corresponds to $d_{\gamma_{1} \ldots \gamma_{n}}(S)$ under $f_{1}$ ). We note that $d_{\gamma_{1} \ldots \gamma_{n}}(B)$ is a 1-cell and we require $T_{0}$ to map the other end of $d_{\gamma_{1} \ldots \gamma_{n}}(B)$ (i.e., the vertex $\neq d_{\gamma_{1} \ldots \gamma_{n}}(A)$ ) onto the other end of $d_{\gamma_{1} \ldots \gamma_{n}}(U)$. Hence $T_{0}$ is defined on the 0 -skeleton of $\mathscr{K}(B)$ as an extension of its definition on $\mathscr{K}(A)$.

The extension of $T_{1}$ from the 1-skeleton of $\mathscr{K}(A)$ to the 1-skeleton of $\mathscr{K}(B)$ may be effected by requiring

$$
\begin{aligned}
& d_{\gamma_{1} \ldots \gamma_{n-1}}(A) \leftrightarrow d_{\gamma_{1} \ldots \gamma_{n-1}}(S), \\
& d_{\gamma_{1} \ldots \gamma_{n}}(B) \leftrightarrow d_{\gamma_{1} \ldots \gamma_{n}}(U), \\
& \mathcal{O}_{\gamma_{1} \ldots \gamma_{n-1}}(B) \leftrightarrow \mathcal{O}_{\gamma_{1} \ldots \gamma_{n-1}}(U)
\end{aligned}
$$

correspond as a homeomorphic extension of $T_{0}$, where $\mathcal{O}_{i}(B)$ and $\mathcal{O}_{\gamma_{1} \ldots \gamma_{n-1}}(B)$ are defined similarly to $\mathcal{O}_{i}(U)$ and $\mathcal{O}_{\gamma_{1} \ldots \gamma_{n-1}}(U)$ as in $\$ 4$, (v) and (vi).

To extend $T_{k-1}$ from the $(k-1)$-skeleton of $\mathscr{K}(B)$ to the $k$-skeleton of $\mathscr{K}(B)$, only $k$-cells not contained in $A$ need be considered if $k<n-1$. Any such cell has the form $d_{i_{1} \ldots i_{n-k+1}}(B)$. Its boundary is a $(k-1)$-dimensional sphere on which $T_{k-1}$ is already defined. Since $d_{i_{1} \ldots i_{n-k+1}}(U)$ is an element in the combinatorial $k$-sphere that is the boundary of $d_{i_{1}} \ldots \hat{i}_{\lambda \ldots i_{n-k+1}}(U)$, where $i_{1} \cdots i_{\lambda} \cdots \hat{\imath}_{n-k+1}$ is the subset of $i_{1}, \cdots, i_{n-k+1}$ obtained by deleting $i_{\lambda}$, the homeomorphism from the boundary of $d_{i_{1} \ldots i_{n-k+1}}(B)$ to the boundary of $d_{i_{1} \ldots i_{n-k+1}}(U)$ has an extension mapping $d_{i_{1} \ldots i_{n-k+1}}(B)$ on $d_{i_{1} \ldots i_{n-k+1}}(U)$ [2].

To extend $T_{n-2}$ from the $(n-2)$-skeleton of $\mathscr{K}(B)$ to the $(n-1)$-skeleton of $\mathscr{K}(B)$ it must be noted that not only $(n-1)$-cells of the form $d_{i_{1}, i_{2}}(B)$ need be considered but also the $(n-1)$-cells $a_{i}$ and $\mathcal{O}_{i}(B)$ must enter. Of course $f_{1}$ and thus $T_{n-1}$ is already defined on $\bar{a}_{i}$ and hence we must also define $T_{n-1}$ not only on the "sides" of $b_{i}$ but also on the "bottom" $\left(=\mathcal{O}_{i}(B)\right)$.

Note first that

$$
\begin{aligned}
\mathcal{O}_{i}(U) & =\text { Closure of } F\left(g_{i}\right) \backslash Z\left(h_{i}\right) \\
\left(=F\left(g_{i}\right) \cap F\left(g_{k_{n}+1}\right)\right) & =G_{n-1} \cdots G_{0}\left(h_{i}^{\prime}\right) \backslash \bigcup_{j<i} G_{n-1} \cdots G_{0}\left(h_{i}^{\prime} \cap h_{j}^{\prime}\right) .
\end{aligned}
$$

Each of the (non-null) sets $G_{n-1} \cdots G_{0}\left(h_{i}^{\prime} \cap h_{j}^{\prime}\right)$ is an $(n-1)$-element whose boundary meets the boundary of $G_{n-1} \cdots G_{0}\left(h_{i}^{\prime}\right)$ in an $(n-2)$-element and hence, by Corollary $14: 5 \mathrm{~b}$ of $[2], \mathcal{O}_{i}(U)$ is an $(n-1)$-element in an appropriate combinatorial subdivision of $G_{n-1} \cdots G_{0}(S)$. Thus $T_{n-2}$ can be extended to carry $\mathcal{O}_{i}(B)$ homeomorphically onto $\mathcal{O}_{i}(U)$.

In general, $T_{k}, k=0, \cdots, n-1$, is a homeomorphism of the $k$-skeleton of $\mathscr{K}(B)$ onto the $k$-skeleton of $\mathscr{K}(U)$ in such a way that, for $k>1, T_{k}$ is an extension of $T_{k-1}$. 
Hence, each $b_{i} \in \mathscr{B}$, which has a boundary that is an $(n-1)$-sphere, is associated with $g_{i}$, whose boundary is the $(n-1)$-sphere $F\left(g_{i}\right)$.

RFMARK. The homeomorphism $T_{n-1}$ carries the $(n-1)$-skeleton of $\mathscr{K}(B)$ onto the $(n-1)$-skeleton of $\mathscr{K}(U)$. If, in the application of the Contraction Axiom, an $m$-fold layer of columnar partitions of $U$ are needed, namely $\mathscr{G}, \mathscr{G}_{1}, \mathscr{G}_{2}, \cdots, \mathscr{G}_{m-1}$, then $B_{1}$ and $\mathscr{G}_{1}$ are produced from $A_{1}$ and $S_{1}$ in the same manner that $B$ and $\mathscr{G}$ were formed from $A$ and $S$.

10. Application of the Contraction Axiom. Since $S \in \mathscr{S}$ and

$$
\mathscr{G}=\left\{g_{1}, \cdots, g_{k_{n}+1}\right\}
$$

is a columnar partition of $U$ (or layer of columnar partitions of sufficiently many layers), there is a homeomorphism $t_{1}$ on $X$, the identity on $X \backslash U$, such that if $g_{i}^{\prime}=t_{1}\left(g_{i}\right)$, then $\operatorname{diam} g_{i}^{\prime}<(\operatorname{diam} U) \eta$. Define

$$
t_{1} T_{n-1}(x)=f_{1}^{\prime}(x), \quad x \in \bigcup F\left(b_{i}\right) .
$$

Then $f_{1}^{\prime}(x)$ maps each $F\left(b_{i}\right)$ homeomorphically on $F\left(g_{i}^{\prime}\right)$ so that certain sets of diameter $<(3 / 4)$ (diameter of $B$ ) are mapped on corresponding sets of diameter $<$ (diameter of $U) \eta$.

The sets $F\left(b_{i}\right)$ and $F\left(g_{i}^{\prime}\right)$ bound regions $B_{i}$ and $U_{i}$ in $B$ and $U$, respectively, and, by the Maximality Axiom, $F\left(g_{i}^{\prime}\right)$ is an element of $\mathscr{S}$, hence the procedure may be iterated. Suppose $B_{i}$ is decomposed into $B_{i j}$ where $j=1, \cdots, k_{n}(i)$. Then $F\left(b_{i j}\right)$ is mapped onto $F\left(g_{i j}\right)$. An application of the Contraction Axiom for $j=1, \cdots, k_{n}(i)$ will transform $g_{i j}$ onto $g_{i j}^{\prime}$ so that diameter $g_{i j}^{\prime}<\eta\left(\right.$ diameter $\left.g_{i}^{\prime}\right)$ $<\eta^{2}$ (diameter $U$ ).

We are supposing $\sigma^{n}$ is a standard $n$-simplex and $\dot{\sigma}^{n}$ its boundary. We imagine that $\sigma^{n}$ is subdivided into a sufficient number of layers of columnar partitions (so that the Axiom of Contraction may be applied to the corresponding partitions of $U$ ),

$$
c_{1}, \cdots, c_{k_{n}} \text { and } \sigma_{1},
$$

where each $c_{i}$ and $\sigma_{1}$ have a diameter $\leqq(3 / 4)\left(\right.$ diameter $\left.\sigma^{n}\right)\left({ }^{2}\right)$.

By the Contraction Axiom for $\mathscr{S}$, a homeomorphism $t$ of $\bar{U}$ on $\bar{U}$, the identity on $S$, is defined so that each of $g_{1}^{\prime}, \cdots, g_{k_{n}}^{\prime}$ and $U_{1}^{\prime}$ have a diameter $<\eta($ diameter $U$ ).

Suppose for each integer $m=1,2,3, \cdots$ there is a subdivision of $\sigma^{n}$ into sub-cells $\sigma^{n}\left(\alpha_{1}, \cdots, \alpha_{m}\right)$ whose interiors partition $\sigma^{n}$ and a subdivision of $U$ into disjoint open sets furnishing a partitioning of $U$

$$
U\left(\alpha_{1}, \cdots, \alpha_{m}\right)
$$

such that the sets of a collection of $\sigma^{n}\left(\alpha_{1}, \cdots, \alpha_{m}\right)$ have a non-null intersection of their closures if and only if the sets of the corresponding $U\left(\alpha_{1}, \cdots, \alpha_{m}\right)$ have a non-null intersection of their closures.

(2) The notation has been simplified here to correspond to a 1-fold layer (see §5). 
Suppose further that as $m$ increases indefinitely the maximum of the diameters of $\sigma^{n}\left(\alpha_{1}, \cdots, \alpha_{m}\right)$ and the maximum of the diameters of $U\left(\alpha_{1}, \cdots, \alpha_{m}\right)$ approach zero. That such subdivisions of $\sigma^{n}$ and $U$ do exist is, of course, the content of the preceding sections.

If $\alpha_{1}, \alpha_{2}, \alpha_{3}, \cdots$ is any sequence of integers such that

then

$$
x=\bigcap_{m=1}^{\infty} \overline{\sigma^{n}\left(\alpha_{1}, \cdots, \alpha_{m}\right)},
$$

$$
h(x)=\bigcap_{m=1}^{\infty} \overline{U\left(\alpha_{1}, \cdots, \alpha_{m}\right)}
$$

defines a unique $y \in \bar{U}$. Clearly $h$ is $1-1$ and onto. The continuity of $h$ follows quickly in this way: For each $m$ let $\operatorname{St}_{\sigma}(x, m)$ denote the union of all $\sigma\left(\alpha_{1}, \cdots, \alpha_{m}\right)$ that have closures meeting $x$; a similar meaning is attached to $\operatorname{St}_{v}(y, m)$. Given $\varepsilon>0$, an integer $m$ is found so that $h(x) \in \mathrm{St}_{U}(h(x), m) \subset S(h(x), \varepsilon)$. But for the same $m, x^{\prime} \in \mathrm{St}_{\sigma}(x, m)$ implies $h\left(x^{\prime}\right) \in \mathrm{St}_{u}(h(x), m)$. Since $\mathrm{St}_{\sigma}(x, m)$ is a neighborhood of $x$ in $\sigma$, this establishes the continuity of $h$ and, since $\sigma$ is compact Hausdorff, that of $h^{-1}$.

This completes the proof that a compact metric space $X$ that admits a family $\mathscr{S}$ described above is an $n$-sphere. To complete the proof of the Theorem (\$7) it is shown in the next chapter that $S^{n}$ has such a family $\mathscr{S}$.

(To consider the locally euclidean case only changes very similar to those of $\S 8$ of [6] need be made.)

\section{CHAPTER III}

11. The family $\mathscr{S}$ in $S^{n}$. In this chapter the converse of the theorem given above is established. That is, if $X$ is topologically $S^{n}$ there exists a family $\mathscr{S}$ of topological $(n-1)$-spheres that satisfy Axioms 1-5.

Let $\mathscr{S}$ denote the collection of topological $(n-1)$-spheres in $S^{n}$ each member of which has a shell neighborhood.

Axiom 1 is fulfilled by virtue of the fact that the Jordan-Brouwer theorem holds in $S^{n}$. Axiom 2 is verified by introducing coordinates in $U$ so that $S$ becomes the unit sphere and making appropriate radial projections from the center of $U(3)$. Axiom 5 is valid since the property of possessing a shell neighborhood is an invariant under homeomorphisms of $S^{n}$ on $S^{n}$.

Axiom 4 is verified as follows. Take $\eta=1 / 4$. By a result of Brown [4], $\bar{U}$ is a closed $n$-cell. Let $h$ map $\bar{U}$ onto a unit ball. The sphere $h(S)$ is given a simplicial subdivision $\mathscr{K}$ so that for $\sigma \in \mathscr{K}, h^{-1}(\sigma)$ has a diameter less than $(1 / 4)$ (diameter of $U$ ). Let $\mathscr{L}$ be isomorphic to $\mathscr{K}$ and let $\mathscr{L}$ lie on such a small sphere $\Sigma$ about the center of $h(\bar{U})$ that if $W$ is the region in $h(\bar{U})$ bounded by $\Sigma$, then diameter

(3) That such coordinates may be introduced is the content of [4]. 
$h^{-1}(W)$ is less than $1 / 4$ times the diameter of $U$. Now one inserts sufficiently many layers in $h(\bar{U})$ so that each cell bounded by a sphere (that projects onto a simplex of $\mathscr{K}$ or $\mathscr{L}$ ) has an image under $h^{-1}$ of diameter $<(1 / 4)$ (diameter $U$ ). This dictates how many layers may be inserted in the columnar partition. Call this partition $\mathscr{G}_{1}$. Note that mesh $\mathscr{G}_{1}<\eta($ diameter $U)=(1 / 4)($ diameter $U)$. Let $\mathscr{G}$ be an arbitrary columnar partition generated by $h^{-1}(\mathscr{K})$ with at least as many layers as $\mathscr{G}_{1}$ such that $F(g) \in \mathscr{S}$ for each $g \in \mathscr{G}$. It may be assumed, by inserting more layers to $\mathscr{G}_{1}$ if necessary, that $\mathscr{G}$ and $\mathscr{G}_{1}$ have the same number of layers. A repeated use of property (vii) at the end of $\$ 5$ gives a homeomorphism of the $(n-1)$-skeleton of $\mathscr{G}$ onto that of $\mathscr{G}_{1}$. Since each element of $\mathscr{G}$ and $\mathscr{G}_{1}$ is in $\mathscr{S}$, this can be extended to the desired homeomorphism $t$.

Axiom 3 may verified as follows. Given two disks, each a subset of a member of $\mathscr{S}$, and that the disks and corresponding spheres meet in an appropriate way, an element of $\mathscr{S}$ must be exhibited that contains the union of the disks.

Let $D_{i}$ be an $(n-1)$-disk such that $D_{i} \subset S_{i}, S_{i} \in \mathscr{S}, i=1,2$. Suppose $S_{1} \cap S_{2}$ contains an annulus $A$ with boundary components $S$ and $T$. Further, $D_{1} \cap D_{2}=\partial D_{1} \cap \partial D_{2}=S$. Two cases present themselves.

$\left(1^{\circ}\right) A \cap\left(E^{\prime \prime} \backslash\left(D_{1} \cup D_{2}\right)\right)=\square$.

Then $A \subset D_{1} \cup D_{2}$ and two sub-cases arise according as $T \subset D_{1}$ or $T \subset D_{2}$. Note that since $S=D_{1} \cap D_{2}=\partial D_{1} \cap \partial D_{2}$, we must have one of the sub-cases. Suppose it is the former. Then let $B=D_{1} \cup D_{2}, U_{1}=\operatorname{Int} D_{1}, U_{2}=\operatorname{Int}\left(D_{2} \cup A\right)$. Since $S_{1}, S_{2} \in \mathscr{S}$, the hypotheses of Lemma 4 of [5] are fulfilled, hence $D_{1} \cup D_{2}(=B)=S^{\prime} \in \mathscr{S}$.

$\left(2^{\circ}\right) A \cap\left(E^{n} \backslash\left(D_{1} \cup D_{2}\right)\right) \neq[]$.

If $x \in A \cap\left(E^{n} \backslash\left(D_{1} \cup D_{2}\right)\right)$, there is a neighborhood of $x$ in $A$ that is disjoint to $D_{1} \cup D_{2}$, hence $\left(2^{\circ}\right)$ implies $A \backslash S \subset E^{n} \backslash\left(D_{1} \cup D_{2}\right)$. Then $A \backslash S$ being connected, it must lie entirely in one component of $E^{n} \backslash\left(D_{1} \cup D_{2}\right)$, say the exterior. Since $S$ is an $(n-2)$-sphere lying on each of $S_{1}, S_{2}$ and $S_{i} \in \mathscr{S}$, there are two tubular neighborhoods of $S$ in $E^{n}$ that may be obtained by restriction of the functions describing the shell neighborhoods of $S_{1}, S_{2}$, respectively. Choose one of these, say, $W_{2}=S \times E^{2}$, where $S \times\{0\}$ is identified with $S$, and $W_{2}$ is obtained from the function describing the shell of $S_{2}$. The annulus, which lies on $S_{2}$ (or some sub-annulus satisfying $\left(2^{\circ}\right)$ ), may be obtained $A=S \times I$, where $I$ is an interval in the interior of $E^{2}$ containing $\{0\}$.

By the plane Schoenflies theorem, there is a homeomorphism $t$ of $E^{2}$ on $E^{2}$, the identity on $S^{\prime}=\partial E^{2}$, that maps a prescribed arc, say $I$, in the interior of $E^{2}$ onto any other arc in the interior of $E^{2}$, in particular, an arc $J$ such that $S \times J$ is an annulus in $D_{2}$, one of whose boundary components is $S$. This may be chosen so that $t$ is the identity on the part of $S_{1} \backslash S_{2}$ near $S$. Clearly $t$ has an extension $t^{*}$ to $S \times E^{2}$.

Let $t^{* *}$ map $E^{n}$ on $E^{n}$ where $t^{* *}(p)=p$ if $p \in E^{n} \backslash\left(W_{2}\right)$, while if $p \in W_{2}$ define $t^{* *}(p)=t^{*}(p)$. Since $t^{*}$ is the identity on $\partial W_{2}$, this is a well-defined homeomorphism 
of $E^{n}$ on $E^{n}$ and if $t^{* *}\left(S_{1}\right)=\tilde{S}_{1}$, then $\tilde{S}_{1}$ and $S_{2}$ satisfy the conditions for $D_{1}$ and $D_{2}$ so that the hypotheses of case $\left(1^{\circ}\right)$ are fulfilled. Hence $D_{1} \cup D_{2}=S^{\prime} \in: \mathscr{S}$.

Thus the family $\mathscr{S}$ satisfies the systems of Axioms 1-5.

Among the homology $n$-spheres, the Axiom 4 distinguishes between the homotopy and topological $n$-spheres. It is not known if this is a real distinction for $n=3$, 4. In all dimensions $n \geqq 3$ this axiom distinguishes between homology $n$-spheres and topological $n$-spheres.

\section{APPENDIX}

Proof of (1). First, it is clear $X$ cannot be totally disconnected, for such a space may be expressed as a finite union of arbitrarily small non-null sets that are both open and closed. Suppose $X$ contains three points $a, b$, and $c$ such that $a$ and $b$ lie in a component $C$ of $X$ and $c \notin C$. Then a sufficiently small sphere $S \in \mathscr{S}$ whose interior contains $c$ will not meet $C$ and Property 1 of the members of $\mathscr{S}$ is violated.

Proof of (2). If $X$ is connected but not locally connected, there is a point $p$ and a pair of open sets $U, V$ containing $p$ such that $\bar{V} \subset U$ and a sequence of components of $\bar{U} \backslash V, K_{0}, K_{1}, K_{2}, \cdots$ such that $\lim K_{i}=K_{0}$ and each of $K_{i}$ meets both $F(U), F(V)$. But for any point $x$ of $K_{0}$ not in the boundary of $U$ or $V$, the local basis at $x$ cannot have arbitrarily small members whose boundaries are in $\mathscr{S}$, a contradiction.

\section{REFERENCES}

1. P. S. Aleksandrov, Combinatorial topology. I, Graylock Press, Rochester, N. Y., 1956.

2. J. W. Alexander, The combinatorial theory of complexes, Ann. of Math. 31 (1930), 292-320.

3. R. H. Bing, A characterization of three-space by partitionings, Trans. Amer. Math. Soc. 70 (1951), 15-27.

4. M. Brown, A proof of the generalized Schoenflies theorem, Bull. Amer. Math. Soc. 66, (1960), 74-76.

5. __ Locally flat embeddings of topological manifolds, Ann. of Math. (2) 75 (1962), 331-341.

6. O. G. Harrold, Jr., Locally peripherally euclidean spaces are locally euclidean, Ann. of Math. (2) 74 (1961), 207-222.

7. - Local unknottedness, local peripheral unknottedness and combinatorial structures, Proceedings of the Institute for 3-dimensional Topology, University of Georgia, Athens Ga., Prentice-Hall, Englewood Cliffs, N. J., 1962.

\section{UNIVERSTTY OF TENNESSEE,}

KNOXVILLE, TENNESSEE 